Original Research Paper

\title{
An Upper Bound to the Number of Conjugacy Classes of Non- Abelian Nilpotent Groups
}

\author{
${ }^{1}$ Bilal Al-Hasanat, ${ }^{1}$ Awni Al-Dababseh, \\ ${ }^{1}$ E. Al-Sarairah, ${ }^{1}$ Sadoon Alobiady and ${ }^{2}$ Mahmoud Bashir Alhasanat \\ ${ }^{I}$ Department of Mathematics, Al Hussein Bin Talal University, Ma'an, Jordan \\ ${ }^{2}$ Department of Civil Engineering, Al Hussein Bin Talal University, Ma'an, Jordan
}

\author{
Article history \\ Received: 22-06-2016 \\ Revised: 08-11-2016 \\ Accepted: 23-05-2017 \\ Corresponding Author: \\ Mahmoud Bashir Alhasanat, \\ Department of Civil \\ Engineering, Al Hussein Bin \\ Talal University, Ma'an, \\ Jordan \\ Email: 20petra07@gmail.com
}

\begin{abstract}
The number of conjugacy classes of symmetric group, dihedral group and some nilpotent groups is obtained. Until now, it has not been obtained for all nilpotent groups. Although there are some lower bounds to this value, there is no non-trivial upper bound. This paper aims to investigate an upper bound to this number for all finite nilpotent groups. Moreover, the exact number of conjugacy classes is found for a certain case of non-abelian nilpotent groups.
\end{abstract}

Keywords: Conjugacy Class, Centre, Nilpotent Class

\section{Introduction}

Classifying finite nilpotent groups is a difficult task, though there are some nilpotent groups that have been classified. The classification of conjugacy classes for certain groups such as symmetric groups and dihedral groups have been determined, see (Erdos and Turan, 1968; Pantea, 2004; Banti, 2005; Héthelyi et al., 2011; Al-Hasanat and Abdullah, 2012). In this research, we will denote $c l_{G}$ as the number of conjugacy classes of a group $G$.

The travail group has only one conjugacy class where an abelian group $G$ has $|G|$ conjugacy classes. Therefore, $1 \leq c l_{G} \leq|G|$ for any finite group $G$.

Erdos and Turan (1968) proved that $G, c l_{G}>$ $\log _{2} \log _{2}|G|$ for any finite group $G$. An improved lower bound was obtained by Sherman (1979), he showed that if $G$ is nilpotent of class $c$, then $c l_{G}>c\left(|G|^{1 / \mathrm{c}}-1\right)+1$, which displays the effect of the nilpotency class $c$ on $c l_{G}$.

The number of conjugacy classes was found for finite nilpotent groups in Lopez (1985), it is obtained as a function of the orders of certain subgroups. The conjugacy classes of p-groups, with an abelian subgroup of index $p$, with $|Z(G)|=\left|G^{\prime}\right|$, were studied in (Pantea, 2004). Zapirain (2011) established a lower bound for the number of conjugacy classes of finite nilpotent groups using the group size multiply by a certain constant c. Recently, Ahmad et al. (2012) obtained general formulas to find the number of conjugacy classes of 2-generator $p$-groups of class 2 .
The lack of using only a lower bound for $c l_{G}$ would make the identification of this value a difficult task, especially for groups of big size. Arbitrarily, let $L=$ $c\left(|G|^{1 / c}-1\right)+1$ be the lower bound obtained by Sherman ( $c$ is the nilpotency class of $G$ ), then $L \leq c l_{G} \leq|G|$ (the trivial upper bound $|G|$ will diverge for large size groups).

In this study, a new approach has been followed by a distinct direction. That is to say, all of the previous research obtained lower bounds for the value of $c l_{G}$ or obtained this value for certain groups and not for all nonabelian nilpotent groups. This work aims to improve the ordinary upper bound $|G|$ to another closed one. The estimation carried the calculation of an amount $u$ as a function of the orders of the group $G$ and its centre $Z(G)$, together with the nilpotency class $c$. The replacement of the value of $u$ instead of $|G|$ as an upper bound will limit the value $c l_{G}$ with more convergent boundaries.

The paper is structured as follows. In the next section we fix our notation and establish some preliminary results. In section 3 the definition of the conjugacy classes is given. In section 4 outline our strategy for estimating the number of conjugacy classes. In addition, we prove the main results and display some examples. In the last section, we describe the research results where the research conclusions were given.

\section{Preliminaries}

Our notation is fairly standard. Throughout, all groups are finite. For a group $G, e$ is the identity element and $Z(G)$ denotes the centre of $G$. Recall the commutator 
of $x, y \in G$ is given by $[x, y]=x^{-1} y^{-1} x y$. For any subgroups $A$ and $B$ of a group $G$, the commutator subgroup $[A, B]$ is the group $\langle[a, b] \mid a \in A, b \in B\rangle$. The derived subgroup of $G$ is:

$$
G^{\prime}=[G, G]=\langle[x, y] \mid x, y \in G\rangle
$$

The direct product of the groups $(G, *)$ and $(H, \cdot)$ is $\{(g, h) \mid g \in G, h \in H\}$ and denoted by $G \times H$; with:

$$
\left(g_{1}, h_{1}\right) \cdot\left(g_{2}, h_{2}\right)=\left(g_{1} * g_{2}, h_{1} \cdot h_{2}\right)
$$

follows that $(G \times H, \cdot)$ is a group.

Let $\phi: H \rightarrow \operatorname{Aut}(G)$ be a group homomorphism, the semidirect product of $G$ and $H$ is the group $\{(g, h) \mid g \in G$, $h \in H\}$ denoted by $G \rtimes H$, where the group operation $\star$ is defined by:

$$
\left(g_{1}, h_{1}\right) \star\left(g_{2}, h_{2}\right)=\left(g_{1}^{\phi\left(h_{2}\right)} * g_{2}, h_{1} \cdot h_{2}\right)
$$

The lower central series of a group $G$ is:

$$
G=\gamma_{0}(G) \geq \gamma_{1}(G) \geq \cdots \geq \gamma_{c}(G) \geq \ldots
$$

where, $\gamma_{i}(G)=\left[\gamma_{i-1}(G), G\right]$.

\section{Definition 1}

A group $G$ is called nilpotent if there exists $c$ in the lower central series such that $\gamma_{c}(G)=\{e\}$ and the smallest such value $\mathrm{c}$ is the class of nilpotency.

\section{The Conjugacy Classes}

The elements $x$ and $y$ in $G$ are said to be conjugate, if there exists an element $g \in G$ such that $x=g y g^{-1}$. The set of all $y$ in $G$, which are conjugate to $x$ is the conjugacy class of $x$ and denoted by $C C_{G}(x)$. The conjugacy classes of $G$ are $\left\{C C_{G}(x) \mid x \in G\right\}$ and the order of this set is $\mathrm{Cl}_{G}$.

The conjugacy relation is an equivalence relation. If no distinct elements of $G$ produce the same conjugacy classes, then $c l_{G}=|G|$. Note that $C C_{G}(e)=\{e\}$, therefore $1 \leq c l_{G} \leq|G|$. The equality holds that if $G$ is the trivial group $(G=\{e\})$ then $c l_{G}=1$, or if $G$ is abelian group then $c l_{G}=|G|$. Unfortunately, for groups of large order, these bounds diverge and we observe that the description of $c l_{G}$ becomes very difficult.

\section{The Number of Conjugacy Classes}

It is clear that $C C_{G}(a)=\{a\}$ for all $a \in Z(G)$. Therefore, $c l_{G}=|Z(G)|+\left|\left\{C C_{G}(x) \mid x \in G \backslash Z(G)\right\}\right|$, where the estimation of the second term is very difficult. The next theorem will show an upper bound for these estimations using only three known values, which are; the order of the group, the order of the group centre and the nilpotency class.

\section{Theorem 2}

Let $G$ be a finite group of nilpotency class $c$ and $Z(G)$ be the centre of $G$. Let $u=|G|-r$, where $r=\left\lfloor\frac{|G|-|Z(G)|}{c}\right\rfloor$. Then:

$c l_{G} \leq u$

\section{Proof}

Let $G$ be a group of nilpotency class c and let $n(G)$ denotes the number of conjugacy classes $C C_{G}(x)$ for all $x$ $\in G \backslash Z(G)$. Then:

$$
c l_{G}=|Z(G)|+n(G)
$$

As $G$ acts by conjugation on the set of all elements $x \in G \backslash Z(G)$, then each orbit has length $\geq 2$ and the number of such orbits is $n(G)$. Hence $n(G) \leq \frac{1}{2}(|G|-|Z(G)|)$. If $G$ is an abelian group, then $c$ $=1,|G|=|Z(G)|$ and $n(G)=0$. Therefore, "Inequality 1 " holds with equality. If $G$ is a group of nilpotency class $c>1$, then $1>\frac{c-1}{c} \geq \frac{1}{2}$. Thus:

$$
n(G) \leq \frac{|G|-|Z(G)|}{2} \leq \frac{c-1}{c}(|G|-|Z(G)|)
$$

Using Equation 2. It follows that:

$$
\begin{aligned}
& c l_{G} \leq|Z(G)|+\frac{c-1}{c}(|G|-|Z(G)|) \\
& =|Z(G)|+\left(1-\frac{1}{c}\right)(|G|-|Z(G)|) \\
& |Z(G)|+|G|-|Z(G)|-\frac{|G|-|Z(G)|}{c} \\
& =|G|-\frac{|G|-|Z(G)|}{c} \\
& \leq|G|-\left\lfloor\frac{|G|-|Z(G)|}{c}\right\rfloor
\end{aligned}
$$

The value of $u$ as it illustrated in the previous theorem is $u=|G|-r$, with $r=\left\lfloor\frac{|G|-|Z(G)|}{c}\right\rfloor \leq$ 
$\frac{|G|-|Z(G)|}{c}$. Knowing that, the set $Z(G)$ is a subgroup of $G$, then $|G| \geq|Z(G)|$. In addition, the nilpotency class $c$ is the index of the lower central series of $G$, so $\mathrm{c}$ is strictly positive integer. Therefore, $0 \leq \frac{|G|-|Z(G)|}{c}<G$. This implies that, $0 \leq r<|G|$. Hence, $0<u=|G|-r \leq|G|$.

\section{Example 3}

The following table explains the use of Theorem 2. It should be followed by Table 1 .

All groups in Table 1 are nilpotent groups. Applying Theorem 2 on this table to get the last column, it appears that $c l_{G} \leq u$ for all groups. If $u$ is reduced more, that is $u$ $<|G|-r$, then $u$ will not probably work as an upper bound to $\mathrm{cl}_{G}$ for all groups listed in this table. As the cyclic group $C_{n}$ is an abelian group, then $c l_{G}=|G|=n$. So, if $u$ $<|G|-r=n-0=n$, then $c l_{G}=n>u$, which implies that $\mathrm{u}$ is not an upper bound to $c l_{G}$.

\section{Corollary 4}

Let $G$ be a finite group of nilpotency class 2 . If $\left|G^{\prime}\right|$ $=2$, then:

$$
c l_{G}=\frac{1}{2}(|G|+|Z(G)|)
$$

\section{Proof}

Let $G$ be a finite group of nilpotency class 2, with $\left|\mathrm{G}^{\prime}\right|$ $=2$. Then:

$$
\begin{aligned}
& y^{2} x=y\left[y, x^{-1}\right]\left[x^{-1}, y^{-1}\right] y x \\
& =y\left(y^{-1} x y x^{-1}\right)\left(x y x^{-1} y^{-1}\right) y x \\
& =x y^{2}
\end{aligned}
$$

for all $x, y \in G$ and the order of $[x, y]$ in $G$ divides the orders of $x Z(G)$ and $y Z(G)$ in $G / Z(G)$. So if $n(G)$ is the number of conjugacy classes of all $x \in G \backslash Z(G)$, then each orbit has length exactly 2 and the number of such orbits is $n(G)$.

Hence $n(G)=\frac{|G|-|Z(G)|}{2}$. Then, the claim is followed by using "Equation 2".

\section{Example 5}

The next tables conclude the required estimations for some groups $G$ to show the relation between $u$ and $c l_{G}$ and to demonstrate the use of Corollary 4. It should be followed by Table 2 and 3 .

Table 2 contains only groups of nilpotency class $c=2$ and $\left|G^{\prime}\right|=2$; using Corollary 4 implies that $c l_{G}=u$.
Table 1. Using Theorem 2 to find $c l_{G}$ for some groups of nilpotency class $c$

\begin{tabular}{llllll}
\hline Group Structure & $|G|$ & $|Z(G)|$ & $c$ & $c l_{G}$ & $u$ \\
\hline$C_{5} \times D_{32}$ & 160 & 10 & 4 & 55 & 123 \\
$C_{n}$ & $n$ & $n$ & 1 & $n$ & $n$ \\
$C_{5} \times D_{8}$ & 40 & 10 & 2 & 25 & 25 \\
$D_{128}$ & 128 & 2 & 6 & 35 & 107 \\
\hline
\end{tabular}

Table 2. The estimated upper bound u meets $c l_{G}$ for groups of nilpotency class 2 and $\left|\mathrm{G}^{\prime}\right|=2$

\begin{tabular}{lrrllll}
\hline Group Structure & $|G|$ & $|Z(G)|$ & $\left|G^{\prime}\right|$ & $c$ & $c l_{G}$ & $u$ \\
\hline$C_{4} \times D_{8}$ & 32 & 8 & 2 & 2 & 20 & 20 \\
$C_{8} \rtimes C_{8}$ & 64 & 16 & 2 & 2 & 40 & 40 \\
$C_{16} \times D_{8}$ & 128 & 32 & 2 & 2 & 80 & 80 \\
$C_{11} \times D_{8}$ & 88 & 22 & 2 & 2 & 55 & 55 \\
$C_{32} \rtimes C_{2}$ & 64 & 16 & 2 & 2 & 40 & 40 \\
$C_{2} \times Q_{8}$ & 16 & 4 & 2 & 2 & 10 & 10 \\
$C_{12} \times D_{8}$ & 96 & 24 & 2 & 2 & 60 & 60 \\
\hline
\end{tabular}

Table 3. The estimated upper bound $\mathrm{u}$ for groups of nilpotency class 2 and $\left|G^{\prime}\right| \neq 2$

\begin{tabular}{lrllll}
\hline Group Structure & $|G|$ & $|Z(G)|$ & $c$ & $c l_{G}$ & $u$ \\
\hline$\left(C_{8} \times C_{2}\right) \rtimes C_{8}$ & 128 & 8 & 2 & 44 & 68 \\
$D_{8} \times D_{8}$ & 64 & 4 & 2 & 25 & 34 \\
$C_{9} \rtimes C_{3}$ & 27 & 3 & 2 & 11 & 15 \\
$C_{9} \rtimes C_{9}$ & 81 & 9 & 2 & 33 & 45 \\
\hline
\end{tabular}

Table 3 contains groups of nilpotency class $c=2$ but $\left|G^{\prime}\right| \neq 2$. So we can use only Theorem 2 to bound the number of conjugacy classes. Therefore, $c l_{G}<u$.

\section{Conclusion}

Using the estimated value $u$ will improve the trivial upper bound of $c l_{G}$, which is $|G|$ and make it closed to the exact value for large groups. The value of $u$ depends on $|Z(G)|$ and the nilpotency class $c$ in addition to the main factor $|G|$, this will show the effect of $c$ on $c l_{G}$, which makes $u$ the first bound to use these values. The estimations meet the same value of $c l_{G}$ for all nilpotent groups, in addition to abelian groups (using Groups, Algorithms and Programming software GAP). In addition, in Corollary 4 we can find the exact amount of $c l_{G}$, using as simple as fewer terms and estimations than the usual estimation.

\section{Author's Contributions}

All authors participated in theoretical parts and contributed to the writing of the manuscript.

\section{Ethics}

This article is original and contains unpublished material. The corresponding author confirms that all 
authors have read and approved the manuscript and no ethical issues are involved.

\section{References}

Ahmad, A., A. Magidin and R.F. Morse, 2012. Two generator p-groups of nilpotency class 2 and their conjugacy classes, Publ. Math. Debrecen, 81: 145-166. DOI: $10.5486 /$ PMD.2012.5114

Al-Hasanat, B.N. and I.O.S. Abdullah, 2012. The conjugacy class of symmetric groups. Int. J. Applied Math., 25: 603-607.

Banti, E.A., 2005. Conjugacy classes and finite pgroups. Arch. Math., 85: 297-303. DOI: $10.1007 / \mathrm{s} 00013-005-1398-7$

Erdos, P. and P. Turan, 1968. On some problems of a statistical group-theory. IV. Acta Math. Acad. Sci. Hung., 19: 113-135. DOI: 10.1007/BF01894517
Héthelyi, L., B. Kulshammer and B. Sambale, 2011. Conjugacy classes and characters of finite p-groups. Cummun. Algebra, 39: 657-685. DOI: $10.1080 / 00927871003598723$

Lopez, A.V., 1985. The number of conjugacy classes in finite nilpotent groups. Rend. Sem. Mat. Univ. Padova.

Pantea, C.A., 2004. On the number of conjugacy classes of finite p-groups. Mathematica, 46: 193-203.

Sherman, G.J., 1979. A lower bound for the number of conjugacy classes in a finite nilpotent group. Pac. J. Math., 80: 253-254. DOI: 10.2140/pjm.1979.80.253

Zapirain, A.J., 2011. On the number of conjugacy classes of finite nilpotent groups. Adv. Math., 227: 1129-1143. DOI: 10.1016/j.aim.2011.02.021 